\title{
Going Beyond Hair: Seven Simple Actions We Can Take to Help Prevent Major Illness in Our Hair Transplant Patients
}

David Josephitis, DO Minneapolis, Minnesota, USA drjosephitis@shapiromedical.com

The field of hair transplantation surgery (HTS) is constantly evolving. New developments are continually being made to improve doctor efficacy and overall procedural success. Given the vast array of backgrounds from which HTS specialists originate, this is not surprising as each doctor brings his or her unique talents to the larger HTS community. Over the years, we have seen contributions to our practice from specialists ranging from dermatology and plastic surgery to family and emergency medicine. Obviously, regardless of background, our focus is the replacement of the follicle. However, as physicians first, we cannot afford to overlook the patient as a whole. By leveraging our varied backgrounds to foster a broader perspective during hair transplantation procedures, we can greatly increase the overall care of our patients while simultaneously enriching our reputation as HTS specialists.

This article is a review of the current literature as it pertains to the preventative health maintenance of HTS patients. Many physicians assume that it is neither their responsibility nor within their scope of practice as specialists to consider the basic health needs of HTS patients any more than it affects their ability to perform a safe and effective hair transplant procedure. Though understandable, this position is shortsighted. Even in the practice of HTS, a field primarily focused on improving the outward appearance of the patient, we understand implicitly that underlying the desire in many patients for hair transplantation is the perceived benefit to psychological and mental health. Let us not miss the body for the hair! When symptoms outside the realm of HT are observed that might compromise a patient's overall health, we are remiss to ignore them.

This article will focus on seven aspects of a patient's medical care outside the traditional scope of HTS that we are ideally suited to handle. There may be other aspects that are not covered here that may be addressed if you feel competent and willing to do so. This is not a step-by-step guide to preventative care in an HTS office. Nor is it intended to "bog down" an already busy practice. It is only meant as a refresher with current recommendations pertaining to the items physicians already know they should be monitoring in their patients. It is recognized in this age of increased liability and decreased scope of specialty that it takes an open mind and a willing desire to intercede in areas outside our usual daily practice. Yet, as physicians, we are first healers and only later specialists. In the end, our patients' lives as well as the status of the HTS specialty as a whole will be improved.

\section{Annual Physical Exam}

The majority of HTS patients are males between the ages of 45 and 60. Differing from female patients who often undergo well-woman exams on a yearly basis, many male patients have not seen a medical professional in years. HTS surgeons may be the only contact these patients have with a medical professional at all. This gives a unique opportunity to affect not only the cosmetic health of these patients, but quite possibly, their overall medical health as well.
A few simple questions can be asked to ascertain if the patient is being cared for by a primary care physician (PCP). Patients (especially those who are middle age and older) who have no PCP and have not been to a physician in years can sometimes be spotted during the HTS history by their nonexistent and "healthy" medical and surgical records. If the patient has not seen a doctor in a few years, a yearly checkup should be advised. For those other patients who have never been to a doctor, a simple recommendation to get a baseline exam can be of great benefit to rule out any serious and potentially treatable diseases.

Of the seven areas discussed, this alone may be the single most important. The HTS history and physical is severely limited when it comes to evaluating the overall health of a patient. Therefore, by putting the patient in touch with a health care provider and emphasizing the importance of routine checkups, an HTS surgeon can help place the patient on track to a healthier life.

\section{Hypertension}

Hypertension (HTN) is a vital sign that can often cancel an HTS procedure if it is outside the "safe" zone. With a chronic elevation in blood pressure (BP), there is an ever-increasing risk of stroke and coronary disease in untreated patients under the age of 65. Currently, patients are advised to have a goal of $<140 /<90$ $\mathrm{mmHg}$, and for some patients with additional comorbidities, even lower BP levels are advised., ${ }^{1,2}$

Often, patients who have histories of HTN but have not followed up with their PCP end up in the office ready for surgery. Each HTS surgeon has his or her own comfort level when it comes to BP and undertaking a safe and successful HT procedure. Physicians are commonly apt to attribute elevations in BP to stress alone. They may mistakenly attribute BP elevations to stressful morning commutes or to "White Coat HTN," which is an elevation in BP only seen in the clinical setting and that affects BP much less than one might think. Studies have shown that when a patient has an elevation in BP secondary to White Coat HTN, there is only a mean elevation of 15 and $7 \mathrm{mmHg}$ in the systolic and diastolic BP, respectively. Furthermore, there is a very low likelihood that a patient with an office diastolic BP $\geq 105 \mathrm{mmHg}$ has White Coat HTN. ${ }^{3}$

If the patient's BP is very high, canceling the HTS may be appropriate. Instead of simply referring the patient back to his PCP for treatment, the physician might be able to take a moment to discuss the importance of BP monitoring. Even if a BP is only mildly elevated, speaking as a physician may motivate patients to get the help they need to improve their health.

\section{Prostate Cancer}

Physicians will undoubtedly encounter patients taking finasteride or desiring to start it for help in the prevention of native hair loss. Patients should be advised of both the benefits as well as the potential side effects. A discussion of prostate specific antigen (PSA) levels and the potential for prostate cancer may also ensue. Prostate cancer is a very common disease diagnosed 
in 242,000 men each year in the United States alone. It is the sixth leading cause of cancer death totaling 28,000 per year. ${ }^{4,5}$ During the patient history, questions regarding a family history of prostate cancer or other prostate disease should be asked in order to determine potential risk factors. The patient should be advised of the alterations in PSA levels that can occur when being tested by a PCP.

As well as advising patients about taking or continuing finasteride, this is an opportunity for the physician to discuss whether getting a PSA level would serve any preventative health benefits. Studies have indicated that an elevation in PSA levels is often observable prior to the onset of prostate cancer by up to 5 or 10 years. ${ }^{6,7}$ However, there is currently some debate regarding whether the benefits of screening outweigh the harm. Some organizations feel that the morbidity involved with frequent and sometimes unnecessary testing and biopsies is not worth the lives saved. Major organizations such as the American Cancer Society (ACS) and the American Urological Association (AUA) recommend involving men in the decision-making process. The ACS recommends a PSA with or without a digital rectal exam (DRE) beginning at age 50 unless the patient belongs to a higher risk group (e.g., family history, African American, etc.), in which case testing should begin at age 40. At a minimum, the AUA recommends getting a baseline at age 40 and considering both a PSA and a DRE on an annual basis. ${ }^{8}$ In these circumstances, when HT surgeons find themselves in a position to discuss the benefits and risks of PSA testing, patients should be fully informed and potentially referred to their PCPs for testing.

\section{Colon Cancer}

During a review of a patient's past surgeries and procedures, specifically looking for any difficulties in bleeding or healing, a discussion about the patient's last colonoscopy may arise. Colorectal cancer (CRC) is the second most common cancer in the United States, causing over 600,000 deaths worldwide. ${ }^{4,5}$ Despite these statistics, many adults that should be tested are not. Between 2002 and 2010, the percentage of adults aged 5075 who should be screened in the United States only rose from around $50 \%$ to $65 \%{ }^{9}$

In addition to finding valuable information concerning bleeding risks and the patient's ability to tolerate past procedures, this might be an excellent opportunity to recommend a screening measure for CRC. The patient can easily be educated about the incidence and risks of the disease and the benefits of screening. Patients with an average risk, according to the American College of Physicians, should be screened starting at age 50 with a fecal occult blood test (FOBT) annually, flexible sigmoidoscopy every 5 years (with FOBT every three years), or colonoscopy every 10 years. ${ }^{10}$

Furthermore, patients should be questioned about a family history of colon cancer. With a positive family history the need for screening should be reinforced beginning 10 years before the age of the youngest affected relative or at age 45 for African Americans, as recommended by the American College of Gastroenterology. ${ }^{10}$

\section{Tobacco, Alcohol, Drug Use}

In the HT history taking, surgeons can easily forget to question patients about their complete social history. Much may be discussed concerning career and home life, but often overlooked is whether they are smokers, heavy drinkers, or recreational drug users. The physician should probe into these areas more deeply to determine the overall health status of the patient. Cigarette smoking, specifically, is one of the most preventable causes of cardiovascular disease, lung cancer, chronic obstructive pulmonary disease, and death. Over 400,000 U.S. deaths can be attributed to tobacco on a yearly basis. ${ }^{11}$

While many of these social questions should be asked in accordance with the individual physician's comfort level and/or determination of importance for the safety of the HT procedure, information concerning alcohol use and smoking does have current and future benefits for both the patient and the HT surgeon. It has been noted in smokers that there are more post-operative complications as well as delayed wound healing, which directly affects the hair transplant procedure. ${ }^{12}$ The United States Preventative Health Services recommends discussing the patient's tobacco use status at every patient visit. Studies show that a physician's encouragement to quit can help stimulate patients to kick the habit. ${ }^{13,14}$ HTS surgeons should educate patients about the risks of smoking, encourage them to quit, and guide them to their PCPs for further counseling and treatment. Smokers who do quit also reduce their future risk of developing a tobaccorelated disease. ${ }^{15}$

Alcohol abuse and drug use may be a more challenging task to deal with, as there are fewer opportunities to screen a patient for these. Still, alcohol and drug use are among the most frequent causes of preventable death, and they often go unnoticed and consequently untreated by primary care. ${ }^{16,17}$ A physician should be on the lookout for any red flags of addiction or abuse of these substances. If there are clear signs that a patient has a drinking or drug issue, the physician might be able to intervene personally, help him or her to get to know what community resources exist, or refer the patient to a PCP for further treatment.

\section{Skin Cancer}

During the physical exam, HTS surgeons determine the current status of hair loss and develop a treatment plan. The hair and skin is routinely examined carefully for signs of disease and for candidacy for the procedure. A physician should have at least minimal training in recognizing unusual skin attributes. This might lead an HTS surgeon to suspect skin cancers such as basal cell, squamous cell, or melanoma, and to recommend the patient see a dermatologist for further evaluation and possible biopsy.

In the United States, melanoma is the fifth and seventh most common form of cancer in men and women, respectively. It is the most fatal form of skin cancer and continues to become more common as in 2011 more than 70,000 invasive melanomas were diagnosed in the United States. ${ }^{5}$ The earlier lesions are identified and treated, the better the survival rates in melanoma cases. ${ }^{18}$

Even though the area of evaluation in the HT physical exam is primarily located to the head and neck, these are areas of frequent sun exposure and common areas for lesions to develop. Even a cursory exam can uncover areas on the scalp, face, and neck that might be suspect. Questions should be posed about changes to the symmetry, border, color, or size of a lesion. If a suspicious area is noted, the physician can also ask about a family history of skin cancer and highlight the importance of a full body skin exam if warranted. HT should also be deferred until a lesion is appropriately assessed, giving a patient additional impetus to act.

$\Rightarrow$ page 56 
Going Beyond Hair from page 55

\section{Obstructive Sleep Apnea}

The majority of the perioperative phase during an HTS is thankfully quite routine and uneventful. Obstructive sleep apnea (OSA) is quite an unusual diagnosis for a PCP to make because sleep studies are not routinely performed in the office. Nonetheless, OSA is a common disorder that affects approximately $10-25 \%$ of adults and can put them at risk for neurocognitive disorders and other medical conditions, as well as increasing mortality for those with cardiovascular comorbidities. It affects males and females both from the ages of 18 to $65 .{ }^{19,20}$ This is another diagnosis that might be challenging to make unless the right questions are asked.

As HTS surgeons, there is a unique opportunity to occasionally monitor sedated patients who have fallen asleep during the procedure. If apneic or hypopnic episodes are witnessed or loud snoring heard, the physician can follow up with questions concerning history of heart disease or family history of OSA. More specific questions can also be asked about sleeping habits, daytime somnolence, periods of silence followed by loud snoring as reported by their bed partners, poor concentration, morning headaches, and nocturnal angina.

The short "sleep study" during an HT procedure and a few targeted questions typically cannot result in an accurate diagnosis, but they can give an index of suspicion that may be sufficient enough to recommend the patient follow up with a physician.

\section{Further Prevention and Conclusion}

Depending on the HTS surgeon's geographical area, background, specialty, and other variables, additional opportunities for disease prevention beyond the seven considered here may arise. These may include the use of ASA as prophylaxis for secondary prevention of stroke and heart disease, the avoidance of HTS in patients with Body Dysmorphic Disorder (BDD) and appropriate psychiatric referral, the severely depressed patient, and cases of domestic violence, especially in the female patient. The take-away point is that, as physicians, we should keep the whole patient's health in mind, calling upon the entirety of our training to administer the very best care. Not only will this benefit our patients, but also it will raise the entire society of hair restoration surgeons to a higher standard.

\section{References}

1. Kannel, W.B. Elevated systolic blood pressure as a cardiovascular risk factor. Am J Cardiol. 2000; 85:251.

2. Mancia, G., et al. 2007 Guidelines for the Management of Arterial Hypertension: The Task Force for the Management of Arterial Hypertension of the European Society of Hypertension (ESH) and of the European Society of Cardiology (ESC). J Hypertens. 2007; 25:1105.

3. Verdecchia, P., Independent predictors of isolated clinic ("white-coat") hypertension. J Hypertens. 2001; 19:1015.

4. Siegel, R., D. Naishadham, and A. Jemal. Cancer statistics, 2012. CA Cancer J Clin. 2012; 62:10.

5. Jemal, A., et al. Global cancer statistics. CA Cancer J Clin. 2011; 61:69.

6. Gann, P.H., C.H. Hennekens, and M.J. Stampfer. A prospective evaluation of plasma prostate-specific antigen for detection of prostatic cancer. JAMA. 1995; 273:289.
7. Draisma, G., et al. Lead times and overdetection due to prostate-specific antigen screening: estimates from the European Randomized Study of Screening for Prostate Cancer. J Natl Cancer Inst. 2003; 95:868.

8. Greene, K.L., et al. Prostate specific antigen best practice statement: 2009 update. J Urol. 2009; 182:2232.

9. Centers for Disease Control and Prevention (CDC). Vital signs: colorectal cancer screening, incidence, and mortality-United States, 2002-2010. MMWR Morb Mortal Wkly Rep. 2011; 60:884.

10. Qaseem, A., et al. Screening for colorectal cancer: a guidance statement from the American College of Physicians. Ann Intern Med. 2012; 156:378.

11. World Health Organization Report on the Global Tobacco Epidemic, 2011: Warning about the dangers of tobacco. World Health Organization, Geneva 2011. http://whqlibdoc. who.int/publications/2011/9789240687813_eng.pdf (Accessed on September 27, 2011).

12. Mills, E., et al. Smoking cessation reduces postoperative complications: a systemic review and meta-analysis. Am J Med. 2011; 124(2):144.

13. A clinical practice guideline for treating tobacco use and dependence: A US Public Health Service report. The Tobacco Use and Dependence Clinical Practice Guideline Panel, Staff, and Consortium Representatives. JAMA. 2000; 283:3244.

14. Lancaster, T., and L. Stead. Physician advice for smoking cessation. Cochrane Database Syst Rev. 2004; CD000165.

15. Anthonisen, N.R., et al. The effects of a smoking cessation intervention on 14.5-year mortality: a randomized clinical trial. Ann Intern Med. 2005; 142:233.

16. Mokdad, A.H., et al. Actual causes of death in the United States, 2000. JAMA. 2004; 291:1238.

17. U.S. Preventive Services Task Force. Screening and behavioral counseling interventions in primary care to reduce alcohol misuse: recommendation statement. Ann Intern Med. 2004; 140:554.

18. Balch, C.M., et al. Prognostic factors analysis of 17,600 melanoma patients: validation of the American Joint Committee on Cancer melanoma staging system. J Clin Oncol. 2001; 19:3622.

19. Punjabi, N.M., et al. Sleep-disordered breathing and mortality: a prospective cohort study. PLoS Med. 2009; 6:e1000132.

20. Jennum, P., and R.L. Riha. Epidemiology of sleep apnoea/ hypopnoea syndrome and sleep-disordered breathing. Eur Respir J. 2009; 33:907. 